\title{
Correlation of Salivary Cortisol with Stress, Anxiety and Depression in Oral Lichen Planus: A Systematic Review and Meta-analysis
}

\author{
Manjushri Waingade ${ }^{1 \oplus}$, Raghavendra S Medikeri ${ }^{2 \oplus}$, Madhura Mahajan $^{3 \odot}$
}

\begin{abstract}
Aim: The aim of this study was to evaluate and correlate the salivary cortisol levels with depression, anxiety and stress scores in patients with OLP. Background: Oral lichen planus (OLP) is an immunopathological disease where patients often relate the onset and aggravation of symptoms to increased levels of depression/anxiety/stress. Research suggests that salivary cortisol can be considered as a biomarker of stress. Psychosomatic diseases such as OLP validate the role of depression/anxiety/stress so that they can be effectively and comprehensively treated. A comprehensive electronic literature search was performed by using scientific databases MEDLINE, PubMed Central, Directory of Open Access Journal, Cochrane and Google Scholar. Studies included case control/cross-sectional studies in which the detection of salivary cortisol levels of the OLP patients and controls was assessed along with assessment of DAS with questionnaire/scales. Quality assessment was done using modified NewcastleOttawa Quality assessment scale (NOS) and The Joanna Briggs Institute (JBI) Critical Appraisal tool.

Review results: Eleven studies comprising 260 OLP patients and 253 controls were included. The results suggest that the depression/anxiety/stress scores were higher in OLP patients than controls. The anxiety, depression and stress scores showed statistically non-significant correlation with salivary cortisol in OLP.

Conclusion: The psychological factors including stress, anxiety and depression plays a crucial role in pathogenesis of OLP. So, supportive psychological treatment also needs to be considered while treating OLP patients.

Clinical significance: Oral lichen planus (OLP) is a psychosomatic disorder and salivary cortisol is biomarker of stress. The psychological factors including stress, anxiety and depression play a crucial role in pathogenesis of OLP. We reported higher salivary levels of cortisol and higher scores of anxiety, depression and stress in OLP patients compared to controls. Similarly, no correlation between anxiety, depression and stress scores with salivary cortisol is established. Thus, we recommend supportive psychological treatment together with the conventional therapy could increase patients' capability to deal with psychological instabilities that can improve the OLP healing.

Keywords: Anxiety, Cortisol, Depression, Oral lichen planus, Saliva, Stress.

World Journal of Dentistry (2022): 10.5005/jp-journals-10015-1890
\end{abstract}

\section{INTRODUCTION}

Oral lichen planus (OLP) is currently recognized as a multifactorial disease with a complex interplay of genetic and environmental factors including patients' lifestyle, stress as well as anxiety. ${ }^{1-5}$ The prevalence of OLP varies from 0.5 to $4 \%$, seen predominantly in middle-aged and elderly women..$^{6-9}$ It is postulated to result from an abnormal T-cell mediated immune response in which auto-cytotoxic $\mathrm{CD}^{+} \mathrm{T}$ cells trigger apoptosis of oral epithelial cells. ${ }^{7,8,10-12}$

Chronic stress is associated with the activation of the hypothalamic-pituitary-adrenal (HPA) axis as well as with depressed immune function. ${ }^{1,3,7,13,14}$ Various studies have shown that stress and anxiety can be considered as etiological factors in OLP, but there is still controversy concerning the exact mechanism. ${ }^{3,4,8,15,16}$ Accumulating evidence supports clinically relevant interrelations between psychological stressors and onset and progression of chronic diseases. Furthermore, psychological stress and psychiatric illness modify the immunological respo nse. ${ }^{1,15-18}$

Cortisol is considered as a primary end product of the HPA axis because it is widely considered as a biological regulator of adaptation and maintenance to homeostasis in response to psychological and physiological challenges. ${ }^{4,8,17,19}$ The measurement of salivary cortisol is an indicator of free cortisol or biologically active \begin{tabular}{l}
\hline 1,3Department of Oral Medicine and Radiology, Sinhgad Dental \\
College and Hospital, Pune, Maharashtra, India \\
2Department of Periodontics, Sinhgad Dental College and Hospital, \\
Pune, Maharashtra, India \\
Corresponding Author: Manjushri Waingade, Department of Oral \\
Medicine and Radiology, Sinhgad Dental College and Hospital, \\
Pune, Maharashtra, India, Phone: +91 7875044983, e-mail: manju. \\
waingade@gmail.com
\end{tabular}

How to cite this article: Waingade $M$, Medikeri RS, Mahajan M. Correlation of Salivary Cortisol with Stress, Anxiety and Depression in Oral Lichen Planus: A Systematic Review and Meta-analysis. World J Dent 2022;13(1):70-79.

Source of support: Nil

Conflict of interest: None

cortisol. So quantification of salivary cortisol could help in analyzing the activity of HPA axis and thus evaluate the role of psychological factors in the etiopathogenesis of OLP. ${ }^{1,4,6,10,20}$

OLP is associated with significantly increased levels of anxiety, depression, and stress, as well as the development or worsening of lesions during times of emotional stress. ${ }^{2-5,10,14,19-25}$ However, few authors point to controversies in the relationship between OLP and psychological factors. ${ }^{4,8,15,16}$

() The Author(s). 2022 Open Access This article is distributed under the terms of the Creative Commons Attribution 4.0 International License (https://creativecommons.org/licenses/by-nc/4.0/), which permits unrestricted use, distribution, and non-commercial reproduction in any medium, provided you give appropriate credit to the original author(s) and the source, provide a link to the Creative Commons license, and indicate if changes were made. The Creative Commons Public Domain Dedication waiver (http://creativecommons.org/publicdomain/zero/1.0/) applies to the data made available in this article, unless otherwise stated. 
Because psychological disorders are not easily measurable, studies have used psychometric testing to quantify and establish a relationship between OLP and behavioral changes. In recent years, the mental health of patients with OLP has been clinically assessed both with and without psychological questionnaires that are acceptable to patients and have enough sensitivity and specificity in their ability to diagnose such diseases. ${ }^{9,26}$

Numerous studies have postulated the role of psychological factors like stress, anxiety and depression in OLP, but with conflicting results. ${ }^{2,4,8,15,17,19}$ Further, previous systematic reviews have reported higher salivary cortisol levels in OLP patients. ${ }^{9,27}$ However, there was no emphasis on the correlation of salivary cortisol levels with depression, anxiety and stress (DAS) scores. Thus, the aim of this systematic review is to evaluate and correlate the salivary cortisol levels with the DAS scores in OLP patients.

\section{Methodology}

This systematic review and meta-analysis was developed and registered in PROSPERO (registration number: CRD42021226601). The research question was "Is there any correlation between salivary cortisol levels and Depression, Anxiety and Stress scores in patients with oral lichen planus."

- Patient (P): Patients diagnosed with oral lichen planus clinically and/or histopathologically.

- Intervention (I): Analysis of salivary cortisol with assessment of anxiety, depression and stress levels in patients diagnosed with oral lichen planus.

- Comparator (C): Healthy subjects not suffering with any diseases/conditions, without other skin lesions and/or not on any drugs.

- Outcome (O): Estimation of salivary cortisol levels and assessment of anxiety, depression and stress scores in oral lichen planus and controls.

\section{Inclusion Criteria}

Case-control/cross-sectional studies in which the detection of salivary cortisol levels in OLP patients and controls was assessed along with assessment of DAS with questionnaire/scales were included. Also, publications written in English language and available as full text were included.

\section{Exclusion Criteria}

Case series/case reports, animal studies, in vitro studies and studies in unpublished formats and articles not available as full text were excluded.

\section{Search Strategy}

Databases including PubMed/MEDLINE, PubMed central (PMC), Google Scholar, Cochrane and Directory of Open Access Journal (DOAJ) were searched from 1st January 2010 to 21st January 2021. The following MeSH terms were searched in PubMed/MEDLINE, PubMed central (PMC) including: "oral," "lichen," "planus," "psychological factors," "stress," "anxiety," "salivary," and "cortisol." In Google scholar and DOAJ the terms used were "oral lichen planus," and "cortisol."

\section{Data Extraction}

The search and screening process were carried out by 2 independent reviewers (MW, MM). The titles and abstracts of all retrieved articles were screened and irrelevant studies were excluded. Full text of the eligible studies were obtained and thoroughly analyzed as per eligibility criteria (inclusion/exclusion) for future data extraction.

Two examiners (MW and RM) evaluated titles, abstracts and full text and if there was a diverging opinion, the disagreement among examiners was reexamined and decisions were made unanimously. Each reviewer first decided on a study's eligibility for inclusion in the systematic review, based on the reported parameters. It was performed using a customized data extraction form, which included following contents: year of publication, study design, study population, mean age, laboratory method of detecting salivary cortisol, tools for measuring the stress/anxiety/depression and outcomes. Inconsistencies were discussed and resolved between the two authors. Missing or unclear information was sought from the authors of the selected papers.

\section{Risk of Bias Assessment}

Two authors (MW and RM) independently estimated the quality/risk of bias of each included study. Case-control studies were evaluated by modified Newcastle-Ottawa Quality assessment scale (NOS). ${ }^{28}$ Risk of bias was assessed domain wise. The studies were considered as good quality/low risk of bias when for the selection domain $\geq 3$ stars; for comparability domain $\geq 1$ star; and for outcome/exposure domain $\geq 2$ stars were obtained. The studies were considered as fair quality/some concern when for the selection domain $\geq 2$ stars; comparability domain $\geq$ stars; outcome/exposure domain $\geq 2$ stars were obtained. The studies were considered as poor quality/high risk of bias when for the selection domain $\geq 0$ or 1 star; comparability domain 0 star; outcome/exposure domain 0 or 1 star were obtained.

The Joanna Briggs Institute (JBI) Critical Appraisal tool was used to estimate quality of analytical cross sectional studies. ${ }^{29}$ The positive answers of $\leq 49 \%$ were considered as high risk of bias, whereas $50-69 \%$ for moderate risk and low risk of bias was considered when the positive answers were $>70 \%$. The disagreements between two reviewers were resolved with discussion with third reviewer.

\section{Statistical Analysis}

The Review Manager 5.4 (RevMan 5.4, The Cochrane Collaboration, Oxford, United Kingdom) applying the mean difference (MD) and 95\% confidence intervals ( $\mathrm{Cls}$ ) was used with a random/fixed-effects analysis. Forest plots and funnel plots were created to visualize the differences between groups and publication bias. The MD was calculated to estimate the salivary cortisol levels in OLP patients versus controls. The $\mathrm{I}^{2}$ statistics were applied to evaluate heterogeneity between the studies. High heterogeneity was determined if $\mathrm{I}^{2}>50 \%$ and low heterogeneity when $\mathrm{I}^{2}<50 \%$. The random or fixed model was used depending upon high or low heterogeneity found, respectively. The Medcalc meta-analysis software was used to evaluate the correlation between the DAS scores and salivary cortisol. Also, publication bias was assessed among the studies using funnel plot and by Begg's and Egger's tests. The $p<0.05$ was considered statistically significant.

\section{Results}

\section{Literature Search}

Figure 1 depicts the study search process conducted according to the PRISMA guidelines. The initial online database (PubMed/MEDLINE, PMC, Cochrane, DOAJ and Google Scholar) and manual reference of included studies search yielded a total of 122 articles. After initial 


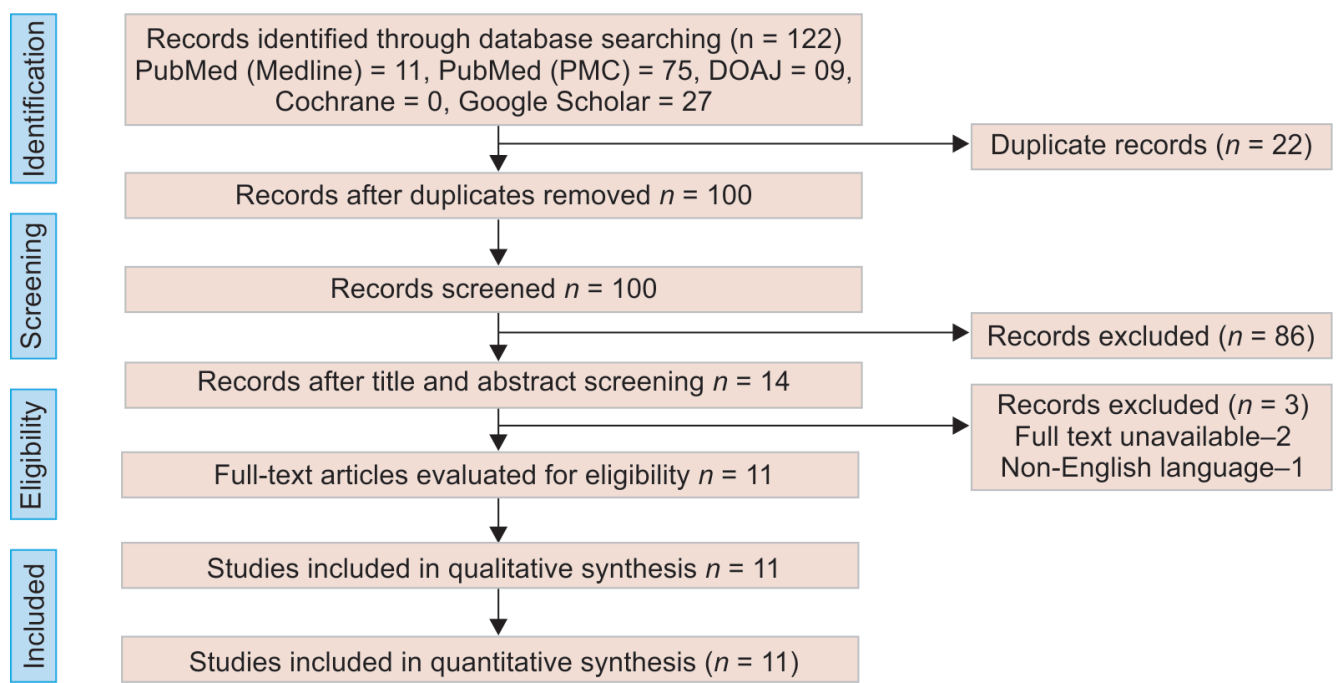

Fig.1: PRISMA flow diagram (2009)

screening and applying the inclusion and exclusion criteria, eleven studies were included in the qualitative and quantitative analysis of salivary cortisol levels in OLP and healthy subjects. ${ }^{1-4,7,8,16,21-24}$

\section{Study Characteristics}

The studies included 260 OLP patients and 253 controls. In all the included studies, ELISA was the most commonly used method of estimation for salivary cortisol level $3,4,8,16,18,20,21$ followed by the chemiluminscence immunoassay in two studies ${ }^{2,7}$ and radioimmunoassay in study by Girardi $C$ et al. ${ }^{1}$

Seven studies had case-control design $n^{1,4,8,16,19,20,22}$ and four studies had comparative cross-sectional design. ${ }^{2,3,7,21}$ Data of all included studies are shown in Table 1.

The scales/questionnaires used for scoring the DAS in various studies were Beck Depression Inventory, Beck Anxiety inventory, Lipps inventory of stress symptoms for adults (LISS) Hamilton anxiety scale, Holms and Rahe's stress scale, the state and trait anxiety inventory, and Percieved stress scale. ${ }^{1-4,7,8,16,18,20-22}$

Girardi et al., ${ }^{1}$ Pires et al., ${ }^{4}$ Pippi R et al., ${ }^{8}$ Gaur B et al., ${ }^{18}$ Gupta and Ahuja. ${ }^{21}$ and Kaur et al. ${ }^{20}$ assessed the DAS scores. Lopez-Jornet et al. $^{2}$ and Vassandacoumara and Daniel ${ }^{2}$ assessed anxiety and depression scores. Nadendla et al. ${ }^{3}$ assessed only the anxiety scores. Zenouz et al. ${ }^{16}$ assessed only stress scores.

The Weighted Kappa ${ }^{a}$ inter-examiner agreement rating was 0.87 with standard error 0.19 and $95 \% \mathrm{Cl} 0.64-1.00$.

\section{Results of Quality Assessment}

All studies showed good and low risk of bias and were rated as high quality. The detail scores of the studies are given in Tables $2 \mathrm{~A}$ and $\mathrm{B}$.

\section{Synthesis of Results}

A quantitative synthesis (meta-analysis) was performed on the selected 11 studies for salivary cortisol levels in OLP and healthy subjects. Four cross sectional studies and seven case control studies were included for assessing the correlation of salivary cortisol levels with anxiety, depression and stress in OLP. ${ }^{1-4,7,8,16,21-24}$

\section{Meta-analysis}

\section{Salivary Cortisol Levels in OLP}

Meta-analysis of cross-sectional and case-control studies showed no difference in the salivary cortisol in OLP and controls (Fig. 2) using random model analysis. The pooled mean difference in cross-sectional studies of the salivary cortisol levels in OLP patients compared with controls was $5.19 \mathrm{ng} / \mathrm{mL}(95 \% \mathrm{Cl}:-0.72,11.10)$ with $\mathrm{I}^{2}=97 \%$ which was statistically non-significant. $(Z=1.72 ; p=0.09)$. Similarly, the pooled mean difference of the salivary cortisol levels in case-control studies was $0.55 \mathrm{ng} / \mathrm{mL}(95 \% \mathrm{Cl}$ : $-2.53,3.63)$ with $\mathrm{I}^{2}=98 \%$ which was statistically non-significant. $(Z=0.35 ; p=0.73)$.

\section{Stress, Anxiety and Depression Scores in OLP and Controls}

Meta-analysis of cross-sectional studies (Fig. 3) in OLP patients compared to controls showed higher anxiety and depression scores which was statistically significant (MD: 7.58; 95\% Cl: 3.06-12.06; $p=0.0009$ and $\mathrm{MD}: 5.39 ; 95 \% \mathrm{Cl}: 2.38-8.40 ; p=0.0005$ respectively). However, high heterogeneity was found in both anxiety $\left(I^{2}=94 \%\right)$ and depression $\left(I^{2}=85 \%\right)$ scores. The stress scores in OLP patients as compared to controls were statistically significant. Quantitative analysis between stress and OLP could not be performed as only one study has been reported.

Case-control studies (Fig. 4) showed higher stress and anxiety scores in OLP as compared to controls which was statistically significant. ( MD: $9.04 ; 95 \% \mathrm{Cl}: 1.31-16.77 ; p=0.02$ and MD: $4.15 ; 95 \%$ Cl: $3.11-5.19 ; p<0.00001$ respectively). No significant differences were observed for depression scores in OLP and controls. (MD: 5.72; $95 \% \mathrm{Cl}:-1.14$ to $12.57 ; p=0.10$ ).

\section{Correlation of Anxiety, Depression and Stress Scores with Salivary Cortisol Levels}

In the two cross-sectional studies (Fig. 5) there was no correlation of anxiety and depression scores with salivary cortisol levels in OLP ( $r$ $=0.0127, p=0.931,95 \% \mathrm{Cl}:-0.267$ to $0.290 ;$ and $r=0.0888, p=0.687$, $95 \% \mathrm{Cl}:-0.331$ to 0.479 respectively) (Fig. 5 ). The Egger's test was significant $(p<0.0001)$ indicating possibility of publication bias. But Begg's test was non-significant $(p=0.3173)$.

In the three case-control studies (Fig. 6), there was no correlation of anxiety, depression and stress scores with salivary cortisol levels in OLP. $(r=0.123, p=0.313,95 \% \mathrm{Cl}:-0.116$ to 0.348 ; $r=0.136, p=0.263,95 \% \mathrm{Cl}:-0.102$ to $0.359 ; r=0.160, p=0.117$, $95 \% \mathrm{Cl}:-0.0403$ to 0.349 , respectively). The Begg's and Egger's test $(p>0.05)$ indicated minimal or nil publication bias across the studies. 


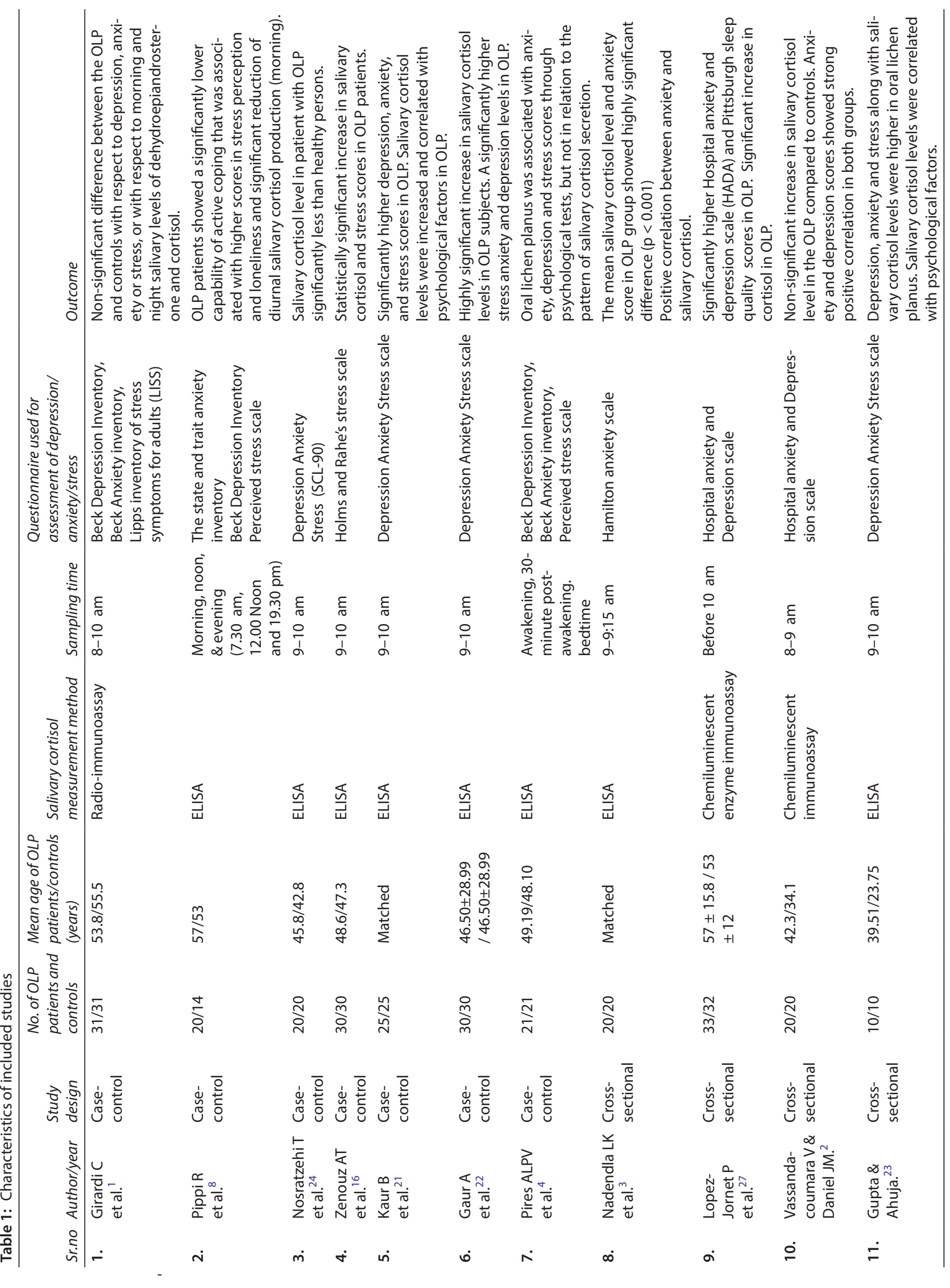


Salivary Cortisol with Stress, Anxiety and Depression in Oral Lichen Planus

Table 2A: Quality assessment of case-control studies using the modified Newcastle-Ottawa Quality assessment scale

\begin{tabular}{|c|c|c|c|c|c|c|c|c|c|}
\hline Studies & Selection & & & & Comparability & Exposure & & & $\begin{array}{l}\text { Total quality } \\
\text { score }\end{array}$ \\
\hline & $\begin{array}{l}\text { Is the case } \\
\text { definition } \\
\text { adequate? }\end{array}$ & $\begin{array}{l}\text { Representative- } \\
\text { ness of the cases }\end{array}$ & $\begin{array}{l}\text { Selection } \\
\text { of controls }\end{array}$ & $\begin{array}{l}\text { Definition } \\
\text { of controls }\end{array}$ & $\begin{array}{l}\text { Comparabil- } \\
\text { ity of cases \& } \\
\text { controls }\end{array}$ & $\begin{array}{l}\text { Ascertainment } \\
\text { of exposure }\end{array}$ & $\begin{array}{l}\text { Same method } \\
\text { of ascertain- } \\
\text { ment for cases } \\
\& \text { controls }\end{array}$ & $\begin{array}{l}\text { Non- } \\
\text { response } \\
\text { rate }\end{array}$ & \\
\hline $\begin{array}{l}\text { Girardi et } \\
\text { al. }^{1}\end{array}$ & 1 & 0 & 1 & 1 & 1 & 1 & 1 & 1 & Good \\
\hline Pippi et al. ${ }^{8}$ & 1 & 0 & 1 & 1 & 1 & 1 & 1 & 1 & Good \\
\hline $\begin{array}{l}\text { Nosratzehi } \\
\text { et al. }{ }^{24}\end{array}$ & 1 & 1 & 1 & 1 & 1 & 1 & 1 & 1 & Good \\
\hline $\begin{array}{l}\text { Zenouz } \\
\text { et al. }^{16}\end{array}$ & 1 & 1 & 0 & 1 & 1 & 1 & 1 & 1 & Good \\
\hline Kaur et al. ${ }^{21}$ & 1 & 1 & 0 & 1 & 1 & 1 & 1 & 1 & Good \\
\hline Gaur et al. ${ }^{22}$ & 1 & 1 & 0 & 1 & 1 & 1 & 1 & 1 & Good \\
\hline Pires et al. ${ }^{4}$ & 1 & 1 & 1 & 1 & 1 & 1 & 1 & 1 & Good \\
\hline
\end{tabular}

Table 2B: Quality assessment of cross-sectional studies using Joanna Briggs Institute (JBI) critical appraisal tool

\begin{tabular}{|c|c|c|c|c|c|c|c|c|c|c|}
\hline \multirow[t]{2}{*}{ Studies } & \multicolumn{8}{|c|}{ Response options* } & \multirow[t]{2}{*}{ Overall appraisal } & \multirow[t]{2}{*}{ Risk of bias } \\
\hline & 1 & 2 & 3 & 4 & 5 & 6 & 7 & 8 & & \\
\hline Nadendla et al. ${ }^{3}$ & Yes & Yes & Yes & Yes & Yes & Yes & Unclear & Yes & Include & Low \\
\hline Lopez-Jornet et al. ${ }^{27}$ & Yes & Yes & Yes & Yes & Yes & Yes & Yes & Yes & Include & Low \\
\hline $\begin{array}{l}\text { Vassandacoumara } \\
\text { and Daniel }\left.\right|^{2}\end{array}$ & Yes & Yes & Yes & Yes & Yes & Yes & Yes & Yes & Include & Low \\
\hline Gupta and Ahuja ${ }^{23}$ & Yes & Yes & Yes & Yes & Yes & Yes & Yes & Yes & Include & Low \\
\hline
\end{tabular}

* 1 - Were the criteria for inclusion in the sample clearly defined?; 2 - Were the study subjects and the setting described in detail?; 3 - Was the exposure measured in a valid and reliable way?; 4 - Were objective, standard criteria used for measurement of the condition?; 5 - Were confounding factors identified?; 6 - Were strategies to deal with confounding factors stated? 7 - Were the outcomes measured in a valid and reliable way?; 8 - Was appropriate statistical analysis used?

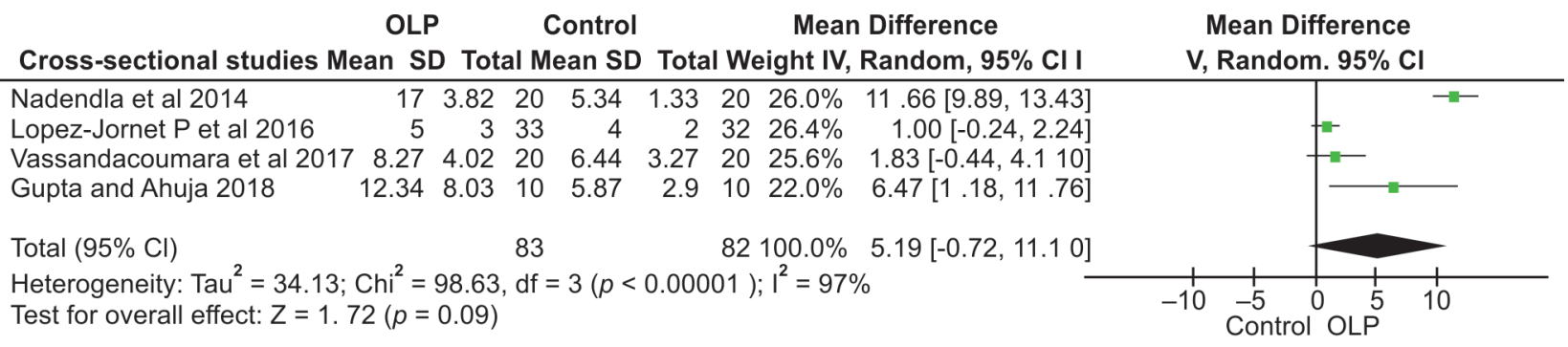

\section{OLP Control Mean Difference Mean Difference}

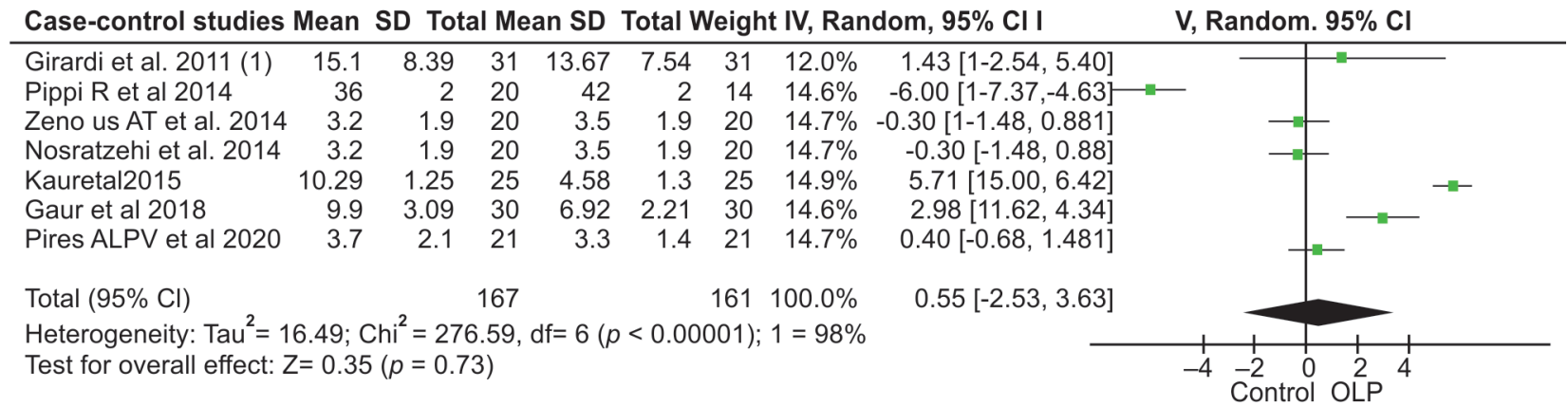

Fig. 2: Forest plot - salivary cortisol levels in the OLP patients vs. controls [cross-sectional studies and case-control studies] 


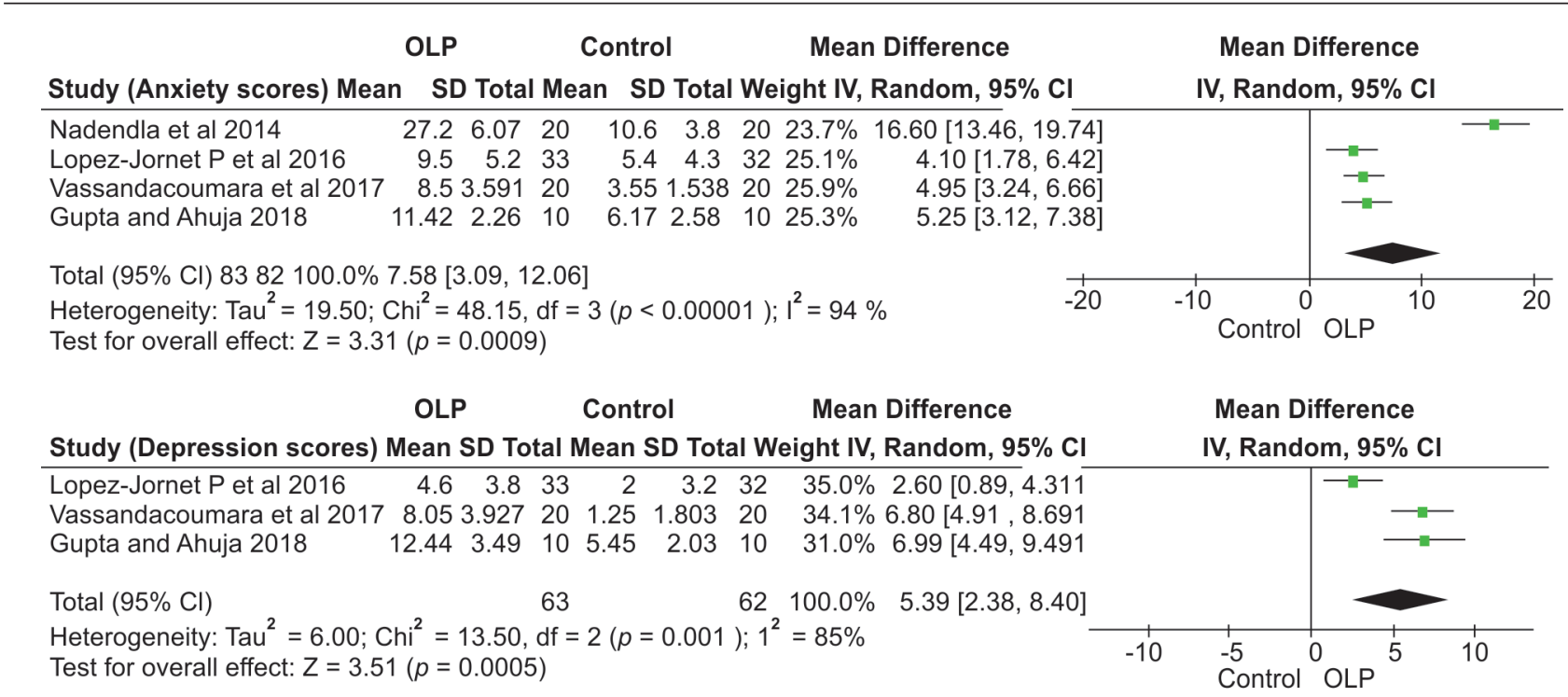

Fig. 3: Forest plot - anxiety and depression scores in the OLP patients vs. controls [cross-sectional studies]

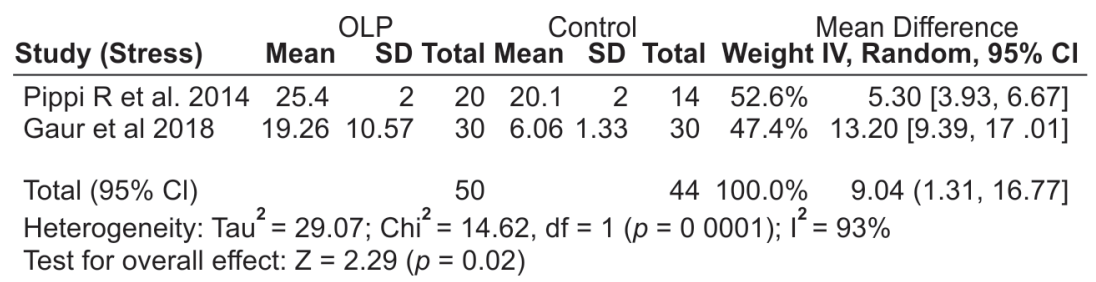

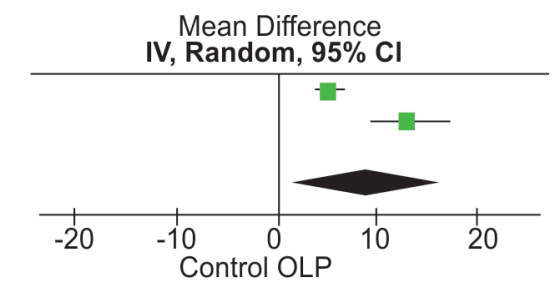

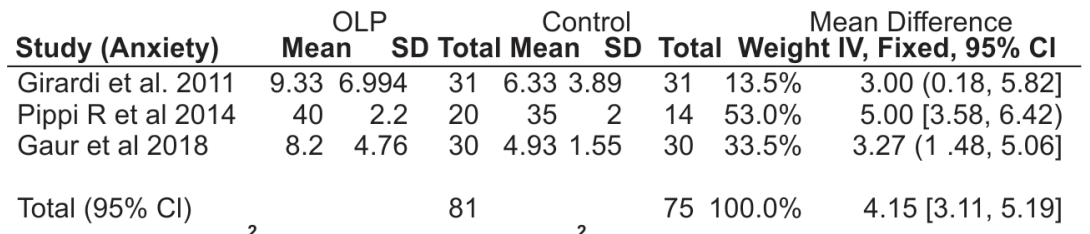

Heterogeneity: $\mathrm{Chi}^{2}=2.94, \mathrm{df}=2(p=0.23) ; \mathrm{I}^{2}=32 \%$

Test for overall effect: $Z=7.85(p=0.00001)$

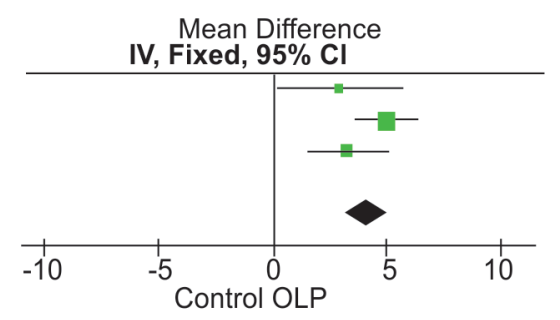

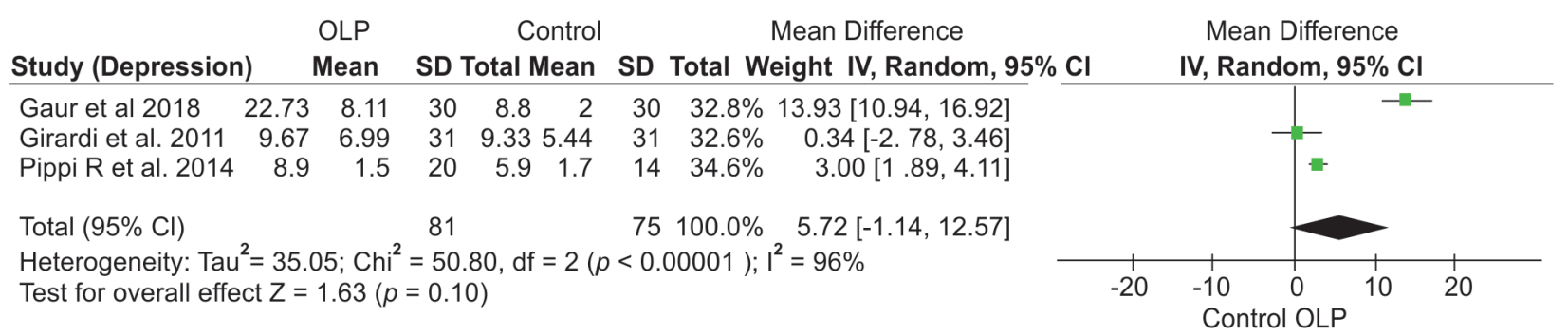

Fig. 4: Forest plot - anxiety and depression and stress scores in the OLP patients vs. controls [case-control studies]

\section{Discussion}

The evidence from this research suggests salivary cortisol levels were not significantly elevated in OLP compared to controls. The anxiety scores in OLP patients were significantly higher in OLP patients as shown in both cross-sectional and case control studies. The depression scores were shown to be higher in OLP patients in cross-sectional studies. The stress scores were also significantly higher in OLP patients as reported in case-control studies. Quantitative evaluation of anxiety, depression and stress scores were statistically not correlated with salivary cortisol levels in OLP patients.

The studies by Gaur et al., ${ }^{18}$ Kaur et al. ${ }^{20}$ and Pires et al. ${ }^{4}$ found a positive correlation between DAS scores with salivary cortisol levels in OLP. Zenouz et al. ${ }^{16}$ found no correlation between stress scores and salivary cortisol. Also, Lopez et al. ${ }^{7}$ and Vassandacoumara et al. ${ }^{2}$ 

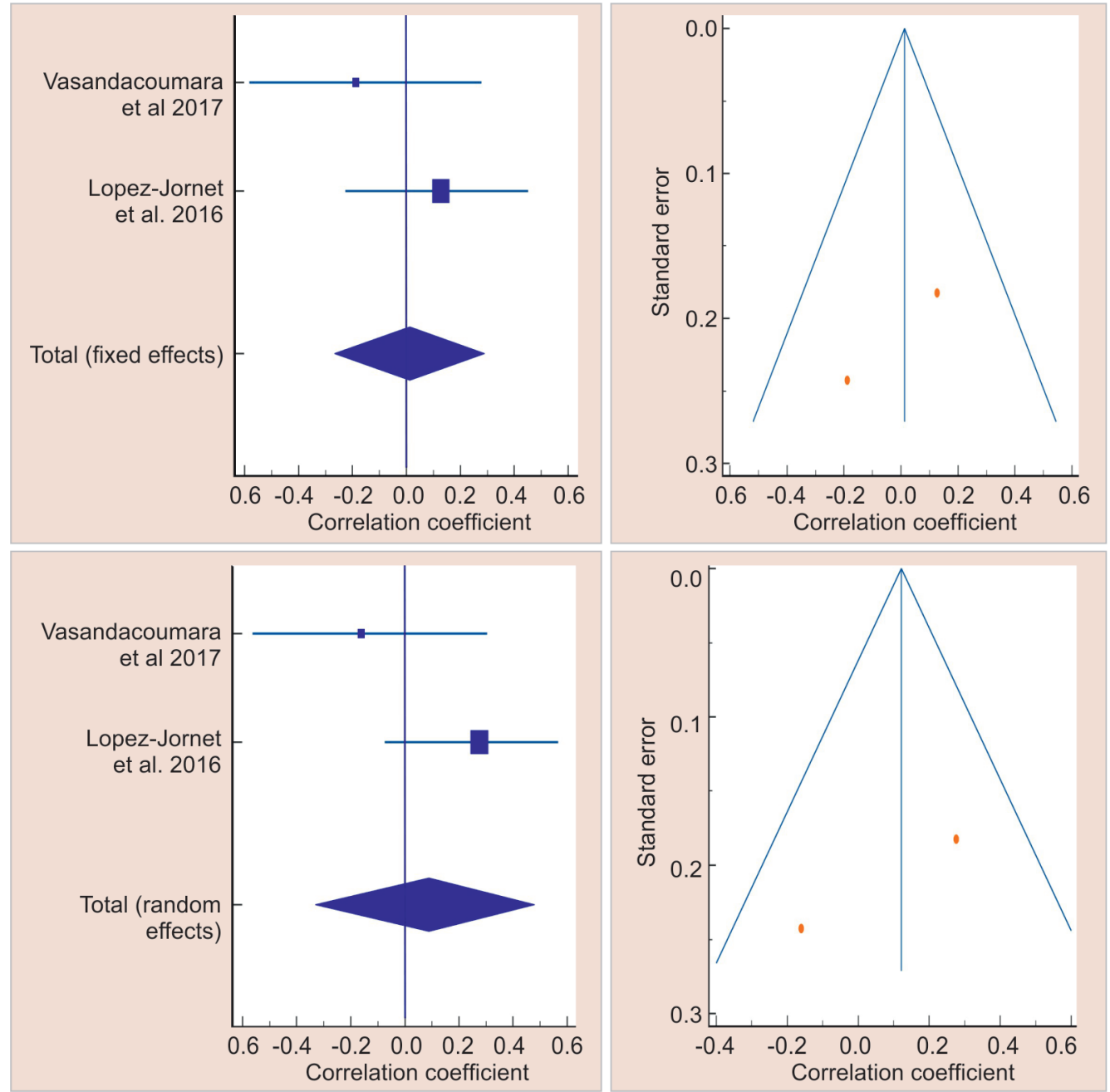

Fig. 5: Forest plot and funnel plot - correlation of anxiety and depression scores with salivary cortisol in OLP patients [cross-sectional studies]

found no correlation between anxiety and depression scores with salivary cortisol levels in OLP. In a study by Nadendla et al., ${ }^{3}$ the outcomes measured were not clearly mentioned as published data was not available although it was stated that cortisol levels were statistically not correlated with anxiety. Hence, it was not included in the meta-analysis. It is evident in the present systematic review that although anxiety, depression and stress score are significantly higher in OLP patients than controls, no correlation between these factors with salivary cortisol levels was observed.

Evidence suggests the role of psychological stress on the immune function that results in the development of psychosomatic disorders. ${ }^{5,18,25,30,31}$ Psychological stress acts as initiating or precipitating factor which causes an imbalance in the Th1/Th2 cytokines primarily. As the repeated episodes of stress continue there is a change or amplification of cytokine production including IL-2. IL-12, IFN- $\gamma$, TNF- $a, \mathrm{IL}-4, \mathrm{IL}-5, \mathrm{IL}-6$, IL-10 and IL-13 that modify the immune response and initiate the inflammatory process which eventually results in the immune dysregulation. Neuroendocrine immune system primarily the catecholamines furthers this process by increasing the function of cytotoxic T cells that result in keratinocyte apoptosis which is the hallmark of OLP. ${ }^{5,32-36}$

This psychological stress leads to activation of HPA axis which leads to increased production of glucocorticoids and catecholamines followed by increased levels of cortisol in the serum and saliva. Many authors have suggested that the salivary cortisol levels indicate the free cortisol that can be used as a non-invasive and simple method for estimation of psychological instabilities. ${ }^{2,5,7,10,20,25,35}$

This meta-analysis has included studies with good study design and analysis indicating high internal and external validity. The risk of bias was performed by two independent examiners for each study. The inter observer agreement was found more than $80 \%$ for studies being assessed.

High heterogeneity was evident in the included studies. This may be attributed to assessment of the DAS scores or variants of OLP in clinical diagnosis. Such variability does not allow creating a standard protocol for assessment of DAS scores in OLP. Since there is no 'standard scale' for measurement of DAS score. Further, the reliability of one scale over another is not estimated. However, the publication bias was observed in this study as evident from the results of Begg's and Egger's test.

Confounding factors that were addressed in most of the included studies are the cortisol awakening response (CAR), which is the rise in cortisol levels from wake time to $30 \mathrm{~min}$ post. In almost all the included studies the timing of salivary cortisol collection was followed as early morning sample to assess this response since 

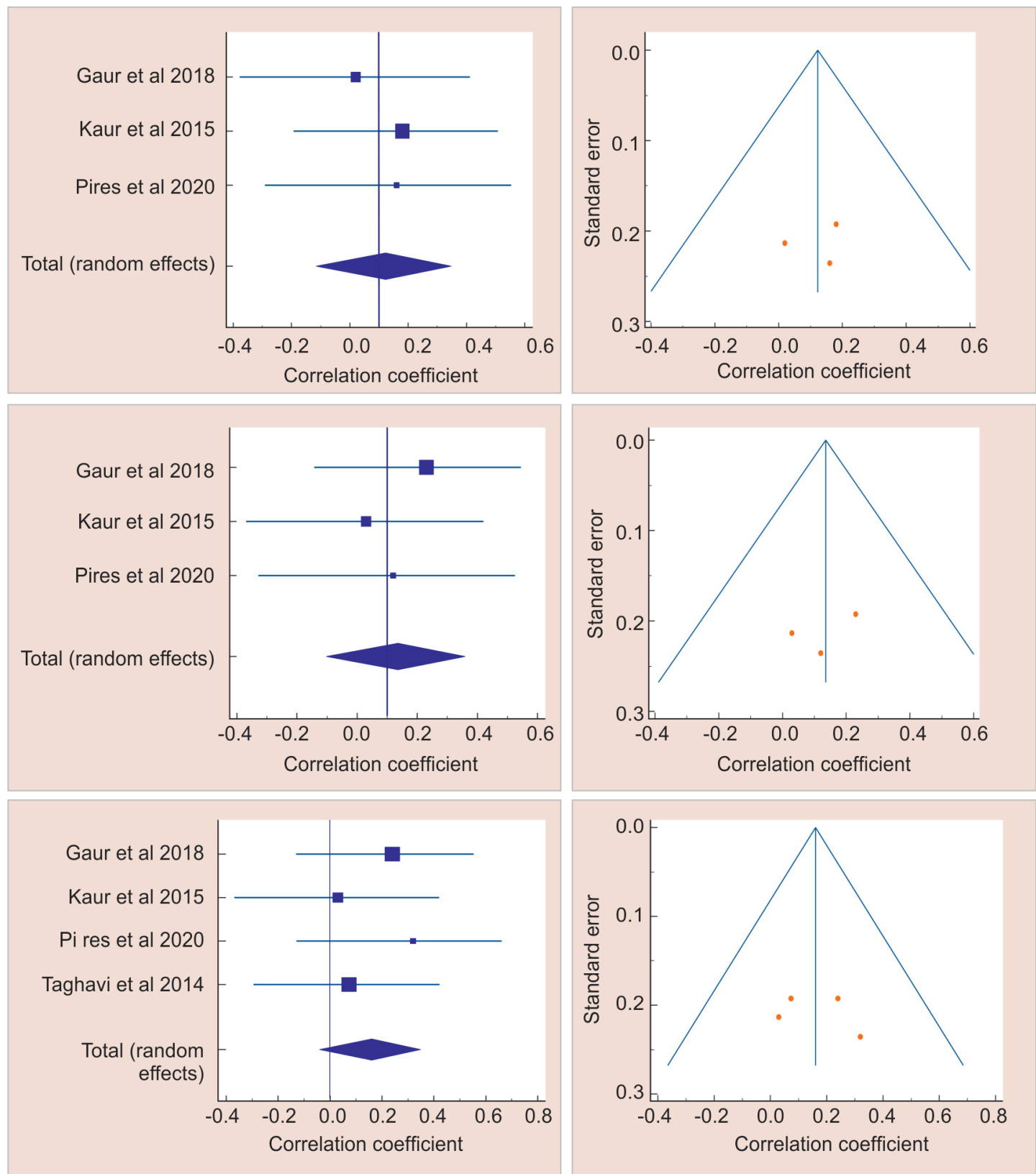

Fig. 6: Forest plot and funnel plot - anxiety and depression and stress scores in the OLP patients vs. controls [case-control studies]

timing of saliva sample collection is crucial in analyzing the results. Also, age and gender related changes associated with the cortisol levels were addressed by age and sex matching the controls in the included studies. Certain diseases and medications could change the cortisol production, so most studies designed strict exclusion criteria for this purpose. Similarly the intake of nicotine, alcohol and smoking could alter the cortisol levels; this issue was addressed by rigid inclusion criteria. ${ }^{37}$

The use of language restriction is one of the inherent bias in this systematic review, providing prejudice in interpreting the evidence. There is currently no agreement on language limits in conducting systematic reviews. Non-English studies may be systematically different from English-language research, and limiting by these features may add a systematic bias as well. Because the review team does not have the ability to read other languages, the decision to employ exclusively English-language publications may be made. Furthermore, the time and expense of translation are not viable given the time and financial constraints. To emphasise, the employment of non-restriction of language barrier would give high findings, and assessment of these studies may undermine the quality assessment in the absence of other inputs. ${ }^{38} \mathrm{~A}$ recent study found no evidence of systematic bias from the use of language constraints in systematic review-based meta-analyses in conventional medicine. ${ }^{39}$

There are certain limitations in the present systematic review that should be taken into consideration. The levels of cortisol secretion vary throughout the day depending on the stressful situations and the concentration of cortisol follows a normal circadian rhythm. ${ }^{37}$ In the present analysis, only two studies have analyzed the diurnal variations of salivary cortisol in OLP 
patients. ${ }^{4,8}$ Also, different scales of assessment of DAS were used in the included studies. ${ }^{3,4,7,8,18,21}$ The difficulty of establishing causality between OLP and psychological factors may be related to the use of different measurement instruments, as well as subjectivity, lack of a methodological standard and the low statistical power of the studies due to small sample size. ${ }^{4,9}$

Nevertheless, the outcome of this meta-analysis should be interpreted carefully considering the high heterogeneity due to methods used to analyze DAS scores and limited number of studies involved for the meta-analysis. More research is required to understand the influence of language restrictions on systematic reviews in specific domains of Medicine. One feasible solution would be to make medical information available in widely accepted languages, preferably English. This will account for the evidence while reducing the potential bias of a language barrier. Future studies are needed with bigger cohorts in order to evaluate all the different variants of OLP. The assessment of stress and circadian rhythm of salivary cortisol also is a field of future research. Moreover, the standardized protocols and scales for assessing the stress, anxiety and depression need to be formulated for better comparison of results.

\section{ConcLusion}

The current systematic review reported no difference in salivary cortisol levels in OLP patients. While higher scores of anxiety, depression and stress in OLP patients were reported compared to controls, but no correlation was seen between the anxiety, depression and stress scores with salivary cortisol levels. However, the results should be interpreted cautiously when correlating salivary cortisol levels with psychosocial factors in OLP since objectively quantifying a psychological disorder can often be difficult and inaccurate because of individuals' subjectivity and the difficulty of methodological standardization. Regardless, we recommend psychological counselling in conjunction with conventional management to help strengthen patients' ability to deal with psychological instabilities that may further improve healing of OLP.

\section{Clinical Significance}

Oral lichen planus is a psychosomatic disorder and salivary cortisol is biomarker of stress. The psychological factors including stress, anxiety and depression plays a crucial role in pathogenesis of OLP. We reported higher levels of salivary cortisol and higher scores of anxiety, depression and stress in OLP patients compared to controls. Similarly, no correlation between anxiety, depression and stress score and salivary cortisol is established. Thus, we recommend psychological counseling in combination with conventional management to help increase patients' capability to deal with psychological instabilities that could further improve OLP healing.

\section{OrCID ID}

Manjushri Waingade ๑ https://orcid.org/0000-0002-1996-573X Raghavendra S Medikeri ๑ https://orcid.org/0000-0002-7879-8644 Madhura Mahajan ๑ https://orcid.org/0000-0001-6470-3096

\section{References}

1. Girardi C, Luz C, Cherubini K, et al. Salivary cortisol and dehydroepiandrosterone (DHEA) levels, psychological factors in patients with oral lichen planus. Arch Oral Biol 2011; 56(9):864-868. DOI: 10.1016/j.archoralbio.2011.02.003
2. Vassandacoumara V, Daniel JM. Correlation between salivary cortisol levels and Hospital Anxiety and Depression scores in oral lichen planus and recurrent aphthous stomatitis. Stomatol Dis Sci 2017; 1:103-108. DOI: 10.20517/2573-0002.2017.08

3. Nadendla LK, Meduri V, Paramkusam G, et al. Association of salivary cortisol and anxiety levels in lichen planus patients. J Clin Diagn Res 2014;8(12):ZC01-ZC03. DOI: 10.7860/JCDR/2014/8058.5225

4. Pires ALPV, Simoura JADS, Cerqueira JDM, et al. Relationship of psychological factors with salivary flow rate and cortisol levels in individuals with oral lichen planus: a case-control study. Oral Surg Oral Med Oral Pathol Oral Radiol 2020;130(6):675-680. DOI: 10.1016/j. oooo.2020.10.004

5. Agha Hosseini F, Sadat Moosavi M, Sadat Sadrzadeh Afshar M, et al. Assessment of the relationship between stress and oral lichen planus: a review of literature. J Islam Dent Assoc Iran 2016;28(2):78-85. DOI: $10.30699 /$ jidai.29.2.78

6. Skrinjar I, Vidranski V, Brzak BL, et al. Salivary cortisol levels in patients with oral lichen planus - a pilot case-control study. Dent J (Basel) 2019;7(2):59. DOI: 10.3390/dj7020059

7. Lopez-Jornet P, Cayuela CA, Tvarijonaviciute A, et al. Oral lichen planus: salival biomarkers cortisol, immunoglobulin $A$, adiponectin. J Oral Pathol Med 2016;45(3):211-217. DOI: 10.1111/jop.12345

8. Pippi R, Patini R, Ghiciuc CM, et al. Diurnal trajectories of salivary cortisol, salivary a-amylase and psychological profiles in oral lichen planus patients. J Biol Regul Homeost Agents 2014;28(1):147-156. PMID: 24750801

9. Cerqueira JDM, Moura JR, Arsati F, et al. Psychological disorders and oral lichen planus: a systematic review. J Invest Clin Dent 2018;e12363. https://doi.org/10.1111/jicd.12363

10. Shah B, Ashok L, Sujatha G. Evaluation of salivary cortisol and psychological factors in patients with oral lichen planus. Indian J Dent Res 2009;20:288-292. DOI: 10.4103/0970-9290.57361

11. Aggarwal A, Agrawal N, Goyal R, et al. Evaluation of psychological factors in patients with oral lichen panus. Annal Dental Special 2020;8(2):5-12.

12. Roopashree MR, Gondhalekar RV, Shashikanth MC, et al. Pathogenesis of oral lichen planus-a review. J Oral Pathol Med 2010;39(10):729-734. DOI: 10.1111/j.1600-0714.2010.00946.x

13. Shetty $\mathrm{P}$, Thopls $\mathrm{L}$, Chatra $\mathrm{P}$, et al. An association between serum cortisol levels in erosive and nonerosive oral lichen planus patients. Webmed Cent Dent 2010;1:WMC0056. DOI: 10.4103/JPBS.JPBS_7_19

14. Chaudhary, S. Psychosocial stressors in oral lichen planus. Aust Dent J 2004;49(4):192-195. DOI: 10.1111/j.1834-7819.2004.tb00072.x

15. Rödström $P O$, Jontell $M$, Hakeberg $M$, et al. Erosive oral lichen planus and salivary cortisol. J Oral Pathol Med 2001;30(5):257-263. DOI: 10.1034/j.1600-0714.2001.300501.x

16. Zenouz A, Mehdipour M, Dadashzadeh $\mathrm{H}$, et al. Evaluation of relationship between salivary cortisol levels and stress intensity in oral lichen planus patients. Der Pharmacia Lettre 2014;6(6):459-461.

17. Karthikeyan $\mathrm{P}, \mathrm{Aswath} \mathrm{N}$. Stress as an etiologic co-factor in recurrent aphthous ulcers and oral lichen planus. J Oral Sci 2016;58(2):237-240. DOI: 10.2334 /josnusd.15-0610

18. Sawant NS, Vanjari NA, Khopkar U, et al. A study of depression and quality of life in patients of lichen planus. Sci World J 2015; 2015:817481. DOI: 10.1155/2015/817481

19. Koray $M$, Dülger $O, A k G$, et al. The evaluation of anxiety and salivary cortisol levels in patients with oral lichen planus. Oral Dis 2003;9(6):298-301. DOI: 10.1034/j.1601-0825.2003.00960.x

20. Ibrahim A, Ibrahem $\mathrm{H}$, Al-Saedy SJ. Cortisol and psychological factors in etiology of Lichen planus. Indian J Forensic Med Toxicol 2020; 14: 1153-1159. DOI: 10.37506/ijfmt.v14i2.3062

21. Kaur B, Sunil MK, Trivedi A, et al. Salivary cortisol in oral lichen planus: a diagnostic marker? J Int Oral Health 2015;7(10):43-48.

22. Gaur A, Kumar CA, Kumar JS, et al. Estimation of salivary cortisol level and psychological assessment in patients with oral lichen planus. J Indian Acad Oral Med Radiol 2018;30:14-17. DOI: 10.4103/jiaomr. jiaomr_98_17 
23. Gupta S, Ahuja N. Estimation of salivary cortisol level in oral lichen planus and oral leukoplakia patients. Int J Health Sci Res 2018;8(6):75-80.

24. Nosratzehi T, Arbabi-Kalati F, Salimi S, et al. The evaluation of psychological factor and salivary cortisol and iga levels in patients with oral lichen planus. Zahedan J Res Med Sci 2014;16(7):31-34.

25. Radwan-Oczko M, Zwyrtek E, Owczarek JE, et al. Psychopathological profile and quality of life of patients with oral lichen planus. J Appl Oral Sci 2018; 26:e20170146. DOI: 10.1590/1678-7757-2017-0146

26. Wiriyakijja P, Fedele S, Porter SR, et al. Patient-reported outcome measures in oral lichen planus: a comprehensive review of the literature with focus on psychometric properties and interpretability. J Oral Pathol Med 2018;47(3):228-239. DOI: 10.1111/jop.12604

27. Lopez-Jornet P, Zavattaro E, Mozaffari HR, et al. Evaluation of the salivary level of cortisol in patients with oral lichen planus: a meta-analysis. Medicina (Kaunas) 2019;55(5):213. DOI: 10.3390/medicina55050213

28. Wells GA, Shea B, O'Connell D, et al. The Newcastle-Ottawa scale (NOS) for assessing the quality of nonrandomized studies in metaanalyses. Ottawa, Canada:The Ottawa Health Research Institute. http://www.ohri.ca/programs/clinical_epidemiology/oxford.htm (accessed 14.04.15)

29. Moola S, Munn Z, Tufanaru C, et al, Mu P-F. Chapter 7: Systematic reviews of etiology and risk In: Aromataris E, Munn Z (Editors). Joanna Briggs Institute Reviewer's Manual. The Joanna Briggs Institute, 2017. Available from: https://reviewersmanual.joannabriggs.org/.

30. Qi M, Gao H, Guan L, et al. Subjective stress, salivary cortisol, and electrophysiological responses to psychological stress. Front Psychol 2016;18(7):229. https://doi.org/10.3389/fpsyg.2016.00229

31. Stojanovich L, Marisavljevich D. Stress as atrigger of autoimmune disease. Autoimmun Rev 2008;7(3):209-213. DOI: 10.1016/j. autrev.2007.11.007
32. Rhodus NL, Cheng B, Ondrey F. Th1/Th2Cytokine ratio in tissue transudates from patients with oral lichen planus. Mediators Inflamm 2007;2007:19854. https://doi.org/10.1155/2007/19854

33. Kalogerakou F, Albanidou-Farmaki E, Markopoulos AK, et al. Detection of Tcell secreting type 1 and type 2 cytokines in theperipheral blood of patients with oral lichenplanus Hippokratia. 2008;12(4):230-235. PMCID: PMC2580045 PMID: 191989

34. Cohen S, Janicki-Deverts D, Miller GE. Psychological stress and disease. JAMA 2007;298(14):1685-1687.DOI: 10.1001/jama.298.14.1685

35. Par M, Tarle Z. Psychoneuroimmunology of oral diseases - a review. Stomatol Edu J 2019;6(1):55-65. DOI: https://doi.org/10.25241/ stomaeduj.2019.6(1).art.7

36. Prolo P, Chiappelli F, Cajulis E, et al. Psychoneuroimmunology in oral biology and medicine. The model of oral lichen planus. Ann NY Acad Sci 2002;966:429-440. DOI: 10.1111/j.1749-6632.2002.tb04244.x

37. Clements AD. Salivary cortisol measurementin developmental research: where do we go from here? Dev Psychobiol 2013;55(3):205-220. DOI: 10.1002/dev.21025

38. McDonagh M, Peterson K, Raina P, et al. Avoiding bias in selecting studies. In: Methods Guide for Effectiveness and Comparative Effectiveness Reviews [Internet]. Rockville (MD): Agency for Healthcare Research and Quality (US); 2008. Available from: https:// www.ncbi.nlm.nih.gov/books/NBK126701/)

39. Morrison A, Moulton K, Clark M, et al. English-language restriction when conducting systematic review-based meta-analyses: systematic review of published studies. Ottawa: Canadian Agency for Drugs and Technologies in Health 2009:1-17. 